\title{
Esophageal Rhabdomyosarcoma
}

National Cancer Institute

\section{Source}

National Cancer Institute. Esophageal Rhabdomyosarcoma. NCI Thesaurus. Code C95623.

A rhabdomyosarcoma arising from the esophageal wall. 